\title{
Symmetry Breaking in the Zero-Energy Landau Level in Bilayer Graphene
}

\author{
Y. Zhao, ${ }^{1}$ P. Cadden-Zimansky, ${ }^{1,2}$ Z. Jiang, ${ }^{3}$ and P. Kim ${ }^{1}$ \\ ${ }^{1}$ Department of Physics, Columbia University, New York, New York 10027, USA \\ ${ }^{2}$ National High Magnetic Field Laboratory, Tallahassee, Florida 32310, USA \\ ${ }^{3}$ School of Physics, Georgia Institute of Technology, Atlanta, Georgia 30332, USA
}

(Received 1 October 2009; published 8 February 2010)

\begin{abstract}
The quantum Hall effect near the charge neutrality point in bilayer graphene is investigated in high magnetic fields of up to $35 \mathrm{~T}$ using electronic transport measurements. In the high-field regime, the eightfold degeneracy in the zero-energy Landau level is completely lifted, exhibiting new quantum Hall states corresponding to filling factors $\nu=0,1,2$, and 3. Measurements of the activation energy gaps for the $\nu=2$ and 3 filling factors in tilted magnetic fields exhibit no appreciable dependence on the in-plane magnetic field, suggesting that these Landau level splittings are independent of spin. In addition, measurements taken at the $\nu=0$ charge neutral point show that, similar to single layer graphene, the bilayer becomes insulating at high fields.
\end{abstract}

DOI: 10.1103/PhysRevLett.104.066801

PACS numbers: 73.63. $-\mathrm{b}, 73.22 .-\mathrm{f}, 73.43 .-\mathrm{f}$

The unique chiral nature of the carrier dynamics in graphene results in a novel integer quantum Hall $(\mathrm{QH})$ effect that distinguishes these atomically thin graphitic materials from conventional two-dimensional systems [1-3]. The nature of these carriers in single layer graphene (SLG) and bilayer graphene (BLG) results in unevenly spaced Landau levels (LL) including a distinctive level located precisely at the particle-hole degenerate zero energy. While the energy spacing of SLG LLs scales as the square root of the LL index $n$ and the square root of the field $B$, BLG levels have energies given by [4] $\epsilon_{n}^{ \pm}=$ $\pm \hbar \omega \sqrt{n(n-1)}$, where $\omega=e B / m$ is the cyclotron frequency with the BLG band mass $m \approx 0.05 m_{e}$, and $n$ is a non-negative integer representing Landau orbit index in each layer. While the valley and spin degrees of freedom lead to a fourfold degeneracy in graphene LLs, the additional orbital degeneracy for the $n=0$ and 1 indices in BLG results in an eightfold degenerate, zero-energy level that is unprecedented in LL physics [3-5].

At high fields, the decreasing radius of the cyclotron orbits gives rise to increasing electron-electron interactions which can perturb the degenerate LLs. Experiments on SLG have demonstrated this high-field symmetry breaking through the appearance of new $\mathrm{QH}$ states at the filling factor sequences $\nu=0, \pm 1, \pm 4$ [6-8]. The precise nature of the field-dependent mechanisms that lift the degeneracies is still under experimental and theoretical debate $[9,10]$. In particular, it has recently been observed that in SLG the $\nu=0 \mathrm{QH}$ state becomes increasingly insulating at higher fields [11,12]. Similar to SLG, the enhanced interactions under high magnetic field are expected to lift the eightfold zero-energy LL degeneracy in BLG. A number of theoretical predictions involving unusual collective excitations have been proposed to occur in this particlehole symmetric LL as its degeneracy is broken [5,13-15].
In this Letter we present transport measurements demonstrating the hierarchy of the splittings for the zeroenergy LL in bilayer graphene as the external magnetic field is increased. Activation energy measurements of these splittings demonstrate energy spacing smaller than the bare electron-electron interaction energy and Zeeman energy. Tilted field measurements indicate that the degeneracy breaking at filling factors above the charge neutral point are independent of spin. While a quantum Hall plateau in the transverse resistance is observed at the charge neutral point, the longitudinal resistance at this point is seen to diverge to an insulating state with increasing field.

The graphene bilayer samples used in this work are deposited on $\mathrm{SiO}_{2}(300 \mathrm{~nm}) / \mathrm{Si}$ substrates by mechanical exfoliation techniques from bulk single crystals [16]. A gate voltage $V_{g}$ is applied to the degenerately doped $\mathrm{Si}$ substrate to control the carrier density $n_{c}$ according to the relation $n_{c}=C_{g}\left(V_{g}-V_{D}\right) / e$, where the areal gate capacitance is $C_{g}=7.1 \times 10^{10} \mathrm{e} / \mathrm{V} \cdot \mathrm{cm}^{2}$ and $V_{D}$ is the gate voltage corresponding to the charge neutrality point. The lower inset of Fig. 1(a) shows an optical microscope image of a typical BLG device used in this study. This device has a mobility as high as $\sim 1 \times 10^{4} \mathrm{~cm}^{2} / \mathrm{V} \cdot \mathrm{s}$ measured at the carrier density $n_{h}=4 \times 10^{12} \mathrm{~cm}^{-2}$. Since the $\mathrm{Au} / \mathrm{Cr}$ electrodes of this device are configured in nonideal Hall bar geometry, the magnetoresistance $R_{x x}$ and Hall resistance $R_{x y}$ were obtained following the van der Pauw method with symmetric $\left(R_{x x}\right)$ and antisymmetric $\left(R_{x y}\right)$ averaging over data from positive and negative magnetic fields. The Hall conductivity $\sigma_{x y}$ is then computed from $R_{x x}$ and $R_{x y}$.

Figure 1(a) shows $R_{x x}, R_{x y}$ (upper inset), and $\sigma_{x y}$. As the backgate voltage alters the carrier density, QH plateaus in $R_{x y}$ with corresponding zeros in $R_{x x}$ are observed. Consequently, the calculated $\sigma_{x y}$ shows well quantized plateau values at $\frac{1}{\nu} \frac{h}{e^{2}}$, where $\nu$ is the integer filling factor. 
(a)

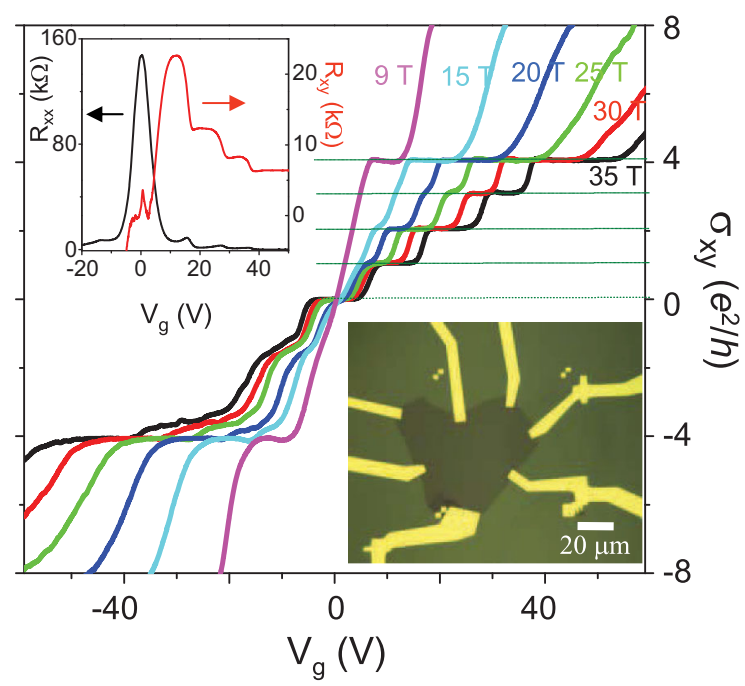

(b)

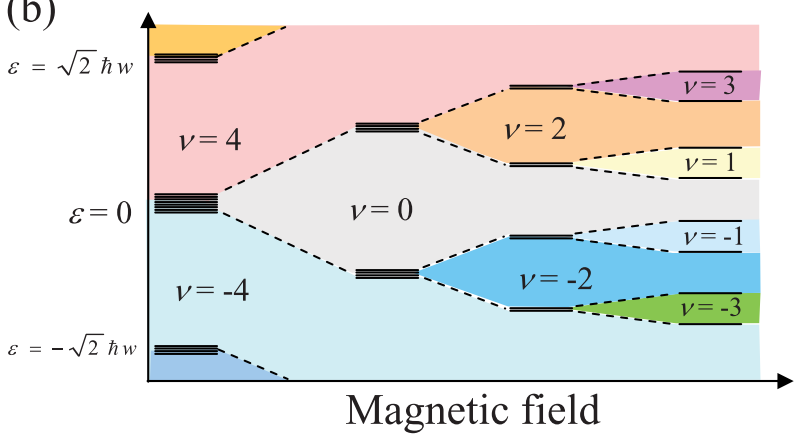

FIG. 1 (color online). (a) Hall conductivity $\sigma_{x y}$, as a function of gate voltage $V_{g}$ at $T=1.4 \mathrm{~K}$ at different magnetic fields: 9, $15,20,25,30$, and 35 T. Upper inset: $R_{x x}$ (in black) and $R_{x y}$ [in gray (red)] as $V_{g}$ varies at $B=35 \mathrm{~T}$. Lower inset: Optical microscope image of a BLG device used in this experiment. (b) Schematic of the zero-energy LL hierarchy in bilayer graphene at high magnetic field.

In the low magnetic field regime $(B<10 \mathrm{~T}), \mathrm{QH}$ states corresponding to $\nu= \pm 4, \pm 8$ are present, as were previously seen in Ref. [3]. As $B$ increases, however, new QH states emerge, as evidenced by additional $\mathrm{QH}$ plateaus at $\nu=0$ and 2 and then $\nu=1$ and 3 at higher fields.

In the high magnetic field regime, $B \geqslant 20 \mathrm{~T}$, the new set of $\mathrm{QH}$ states demonstrates the full eightfold degeneracy lifting in the zero-energy LL. On close inspection of $\sigma_{x y}$ in different magnetic fields, we can construct the hierarchical evolution of these new $\mathrm{QH}$ states. As the field increases from the low magnetic field regime, the $\nu=0$ and 2 states start to develop at $15 \mathrm{~T}$ and are fully evolved by $20 \mathrm{~T}$, while the $\nu=1$ and $\nu=3$ states start to develop at $20 \mathrm{~T}$ and are fully evolved by $25 \mathrm{~T}$. This hierarchical appearance of the new QH states is in accordance with the sequential symmetry breaking of the zero-energy LL degeneracy depicted in Fig. 1(b) and suggests that different symmetry-breaking processes are relevant as $B$ increases.

Mechanisms for breaking the LL degeneracy include disorder, lattice strain, and charged impurities. The mag- nitude of the last of these can be estimated by assuming that the $\sim 2 \mathrm{~V}$ offset of the charge neutral point gate voltage $V_{D}$ from zero energy reflects an aggregate $1.4 \times$ $10^{11} \mathrm{~cm}^{-2}$ charged impurity density on the bilayer. The location of these charge impurities relative to the backgate can affect the opening of the band gap due to the symmetry-breaking perpendicular electric field across the $d=0.34 \mathrm{~nm}$ layer spacing. Assuming these impurities reside on the top layer, an upper bound of such a gap can be estimated to be $9 \mathrm{meV}$ from an unscreened capacitance model. Considering the screening effect [17], however, this valley splitting gap is likely much smaller than this upper bound, close to the same order as the high-field ( $B \gtrsim 20 \mathrm{~T}$ ) bare Zeeman energy. Further quantitative analysis of this energy scale, however, requires experimental quantification of the location of the charged impurities.

In addition to the above mentioned mechanisms, there are two field-dependent factors that can lead to the lifting of the eight degeneracies of this LL: Zeeman splitting and electron-electron interactions. Zeeman splitting is given by $\Delta_{z}=g \mu_{B} B$, where $\mu_{B}$ is the Bohr magneton, and $g$ is the gyromagnetic factor for the carriers in BLG. The Coulomb interactions between electrons are given by $e^{2} / \epsilon l_{B}$, where $\epsilon \sim 4$ is the dielectric constant for graphene and $l_{B}=$ $\sqrt{\hbar / e B}$ is the magnetic length. In order to produce full eightfold symmetry breaking, these mechanisms must lift not only the spin and valley degeneracies, but the $n=0$ and $1 \mathrm{LL}$ orbital degeneracy. Barlas et al. have suggested that the exchange term from Coulomb interactions between

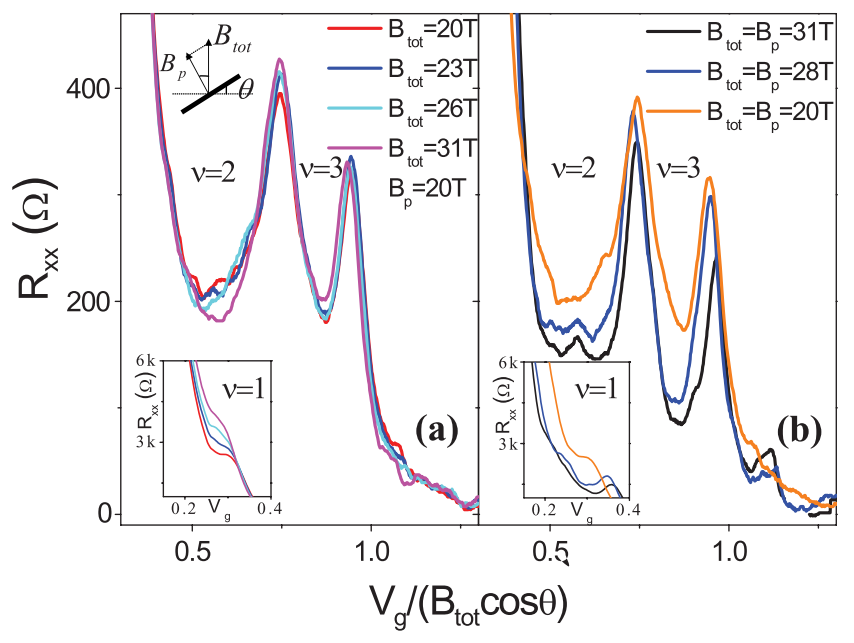

FIG. 2 (color online). (a) Magnetoresistance $R_{x x}$ as a function of normalized backgate voltage $V_{g} /\left[B_{\text {tot }} \cos (\theta)\right]$ around $\nu=2$ and $\nu=3$, at four different total magnetic fields with the same perpendicular field. Upper inset: Schematic diagram of tilted field. Lower inset: $R_{x x}$ vs $V_{g} /\left[B_{\text {tot }} \cos (\theta)\right]$ at $\nu=1$ state under the same condition as in the main figure. (b) $R_{x x}$ as a function of normalized gate voltage with a zero tilting angle at different total fields, with the same scale as (a). Lower inset: $R_{x x}$ vs $V_{g} /\left[B_{\text {tot }} \cos (\theta)\right]$ at $\nu=1$ state. All data are taken at $1.5 \mathrm{~K}$. 
electrons in these degenerate orbital states leads to a $\mathrm{QH}$ ferromagnetic state where the eightfold symmetry is lifted by this exchange interaction. A Hund's rule-like hierarchical symmetry breaking in the zero-energy LL is predicted with this exchange-enhanced Zeeman splitting followed by the spin-independent valley and orbital splitting [5].

In order to test out this hierarchical degeneracy lifting and the role of electron-electron interactions, we first note that sublattice or interlayer splittings that are associated solely with the Coulomb interactions between electrons localized in LL orbits should depend only on the normal magnetic field $\left(B_{\perp}\right)$ but not the in-plane field $\left(B_{\|}\right)$. Whereas spin splitting includes a Zeeman component determined by the total field $B_{\text {tot }}=\sqrt{B_{\perp}^{2}+B_{\|}^{2}}$. Experimentally, this idea can be tested out by measuring the samples in a series of tilted fields where we can examine the $R_{x x}$ minima in different $B_{\perp}$ and $B_{\|}$by tuning the tilting angle $\theta$ and $B_{\text {tot }}$. Figure 2 displays the change of $R_{x x}$ as a function of the gate voltage normalized by $B_{\perp}=B_{\text {tot }} \cos \theta$, in two different experimental conditions.

In Fig. 2(a), we first fix $B_{p}=20 \mathrm{~T}$ and then vary $B_{\|}$. For $\nu=2,3 \mathrm{QH}$ states, $R_{x x}^{\min }$, the minima of $R_{x x}$ corresponding to the $\mathrm{QH}$ zero magnetoresistance, display less than $\sim 10 \%$ of variation, indicating no significant changes are induced by applying $B_{\|}$. For comparison, in Fig. 2(b), we show $R_{x x}$ as a function of $B_{\perp}$ with fixed $\theta=0$; that is, $B_{\|}=0$ and $B_{\perp}=B_{\text {tot }}$. In this case $R_{x x}^{\min }$ for $\nu=2$ and 3 increases by $\sim 40 \%$ as $B_{\perp}$ decreases from 31 to $20 \mathrm{~T}$, a factor of 4 larger change than the change of Fig. 2(a) where $B_{\text {tot }}$ decreases from 31 to $20 \mathrm{~T}$ while $B_{\perp}$ was kept at $20 \mathrm{~T}$. The fact that $R_{x x}^{\min }$ for $\nu=2$ and 3 shows little to no dependence on the in-plane field strongly suggests that both these $\mathrm{QH}$ states are from nonspin origin and thus are due to the breaking of the valley or orbital degeneracy of the zero-energy LL.

As for the $\nu=1 \mathrm{QH}$ state, the experimental data of its tilted field dependence, shown in the insets of Fig. 2, display an increasing $R_{x x}^{\min }$ with either an increasing in-plane field or a decreasing normal field. While this evolution is consistent neither with an electron-electron or spin origin, it is likely that the increasing $R_{x x}^{\min }$ shown in the inset of Fig. 2(a) is due to the proximity of this filling factor to the increasingly insulating behavior of the bilayer at the charge neutrality point, discussed below. However, the emergence of the $\nu=1$ and $\nu=3$ filling factors at similar magnetic fields, along with the expected hierarchy of successive degeneracy breakings schematically shown in Fig. 1(b), suggests that the mechanism underlying the $\nu=1$ breaking should be the same as that for $\nu=3$, i.e., not from a spin origin. We note that a spin-independent mechanism is consistent with theoretical predications of the formation of a spin polarized QH ferromagnet in BLG at high magnetic fields [5,14].

To further understand the nature of the fully lifted LL degeneracy, we determine the energy of the LL splittings by measuring $R_{x x}^{\min }$ at different temperatures $T$.
Figures 3(a)-3(c) show $\log R_{x x}^{\min }$ versus $1 / T$ for the $\nu=$ 1,2 , and 3 states. Since $R_{x x}^{\min } \propto \exp \left[-(\Delta E-2 \Gamma) / 2 k_{B} T\right]$, where $\Delta E$ is the energy gap between two subsequent LLs and $\Gamma$ is the LL energy broadening, the observed Arrhenius behavior in these plots allows us to estimate $\Delta E$ at different fields from the slope of the line fits. Figure 3(d) displays the field dependence of the activation gap for $\nu=1,2$, and 3. Generally, $\Delta E$ increases with increasing $B$ as expected. It is also noted that at given $B$, $\Delta E_{\nu=2}>\Delta E_{\nu=3}, \Delta E_{\nu=1}$, indicating that the even $\nu$ states have larger energy than the odd $\nu \mathrm{QH}$ states in accordance with the LL symmetry-breaking hierarchy of Fig. 1(b). As for the field dependence, we find that a $\sqrt{B}$ fit is better for $\nu=2$ and $\nu=3$ states. Attempts to fit the gap evolution linearly to $B$ result in a positive $y$-axis intercept for the $\nu=2$ and $\nu=3$ gaps and thus a nonphysical negative LL broadening $\Gamma$. From the $y$ intercept of the $\sqrt{B}$ dependence, we can extract a physically reasonable LL broadening of $\Gamma / k_{B}=1.4$ and $3.7 \mathrm{~K}$ for the $\nu=2$ and 3 states, respectively. Although the apparent $\sqrt{B}$ dependence provides an independent confirmation of nonspin origin of the QH states $\nu=2,3$, we note that the observed energy scale $\Delta E$ is much smaller than the Coulomb energy or even the bare Zeeman energy-the energy scales on which the splitting was predicted to occur $[5,14]$. For $B=15 \mathrm{~T}$, the Coulomb energy of $370 \mathrm{~K}$ and the Zeeman energy of $E_{z}=21 \mathrm{~K}$ are both larger than $\Delta E_{\nu=2}=8.6 \mathrm{~K}$ at this field. It is possible that this large energy discrepancy can be accounted for by disorder impurities reducing the transport gap near the charge neutrality point of BLG. A model of screened impurity scattering coupled with the estimate of the impurity density in our

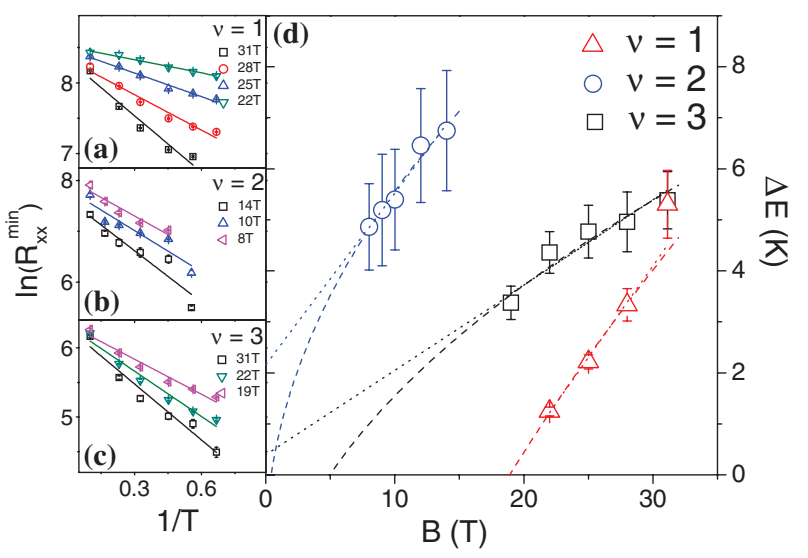

FIG. 3 (color online). (a) Arrhenius plots of $R_{x x}^{\min }$ 's as a function of $1 / T$ at different fields for $\nu=1$ state, the lines with respective colors are the linear fits to the data points. (b) Arrhenius plot for $\nu=2$ state. (c) Arrhenius plot for $\nu=$ 3 state. (d) Energy gap $\Delta E$ versus magnetic field $B$ for different filling factors $\nu=1$ (open red triangle), $\nu=2$ (open green circle), $\nu=3$ (open black square ). The dotted lines are linear fits, whereas the dashed lines are square-root fits. 


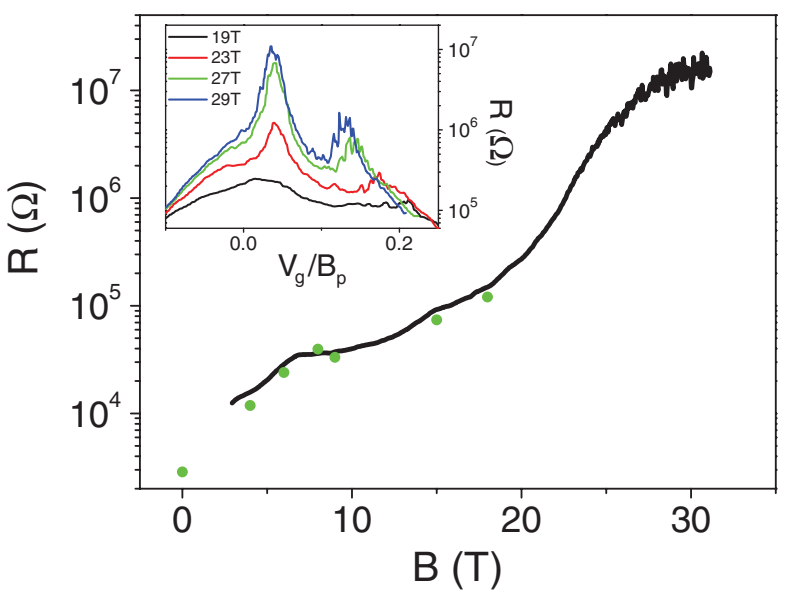

FIG. 4 (color online). Maximum resistance, measured in a 2-probe constant voltage bias method, as a function of magnetic field, which is applied normally to the graphene plane, at a fixed gate voltage $V_{g}=4.1 \mathrm{~V}$ around the charge neutrality point. The green dots are $R_{x x}^{\max }$ 's at $\nu=0$ using a 4-probe measurement. They are taken at lower magnetic fields $(B<20 \mathrm{~T})$ and multiplied by a factor of 4 to match the 2-probe data. Upper left inset: Gate sweep of 2-probe resistance at different fields: 19, 23, 27 , and $29 \mathrm{~T}$ from bottom to top. All data are taken at $1.5 \mathrm{~K}$.

samples yields a disorder energy scale of $\sim 200 \mathrm{~K}$ near the charge neutrality [18].

We finally focus our attention on the $\nu=0 \mathrm{QH}$ state. As with SLG, the presumed $\nu=0$ splitting at the charge neutral point is not directly observable as a zero in $R_{x x}$. Rather, $R_{x x}$ displays a maximum at this point, whose value increases with increasing $B$. In Fig. 4, we display our measurement of $R_{x x}$ at the charge neutrality point in BLG. The measured resistance shows quasiexponential growth as $B$ increases, up to $\sim 10 \mathrm{M} \Omega$ at $30 \mathrm{~T}$. To avoid the self-heating of the graphene discussed in Ref. [12] and to measure resistances $>10 \mathrm{M} \Omega$ we employ a 2-probe ac measurement configuration with a constant voltage bias of $500 \mu \mathrm{V}$, resulting in only $\sim 10 \mathrm{fW}$ of heating at the highest fields. The contact resistance included in this measurement setup is relatively small $(\sim 1 \mathrm{k} \Omega)$, but as an additional confirmation that it does not affect the behavior of the measured resistance, we cross-check the 2-probe measurement using a conventional, current biased, 4-probe measurement at fields low enough that the resistance could be reliably measured with the voltage probes input to an amplifier with $10 \mathrm{M} \Omega$ input impedance. Under the assumption that the contact resistance does not undergo a dramatic change above $20 \mathrm{~T}$, the exponentially divergent $R_{x x}$ behavior at high magnetic field is similar to analogous measurements that have been performed on SLG [11,12], where a field-induced $\mathrm{QH}$ insulator has been proposed. We also note that the gate sweep (inset of Fig. 4) displays a growing number of local maximum in $R_{x x}$, presumably due to the inhomogeneous distribution of these insulating states at high magnetic field [19].
In conclusion, we have observed the full degeneracy lifting of the zero-energy LL in bilayer graphene. Independent measurements of the longitudinal resistance zeros for the newly observed filling factors as a function of perpendicular field and temperature each indicate that the degeneracy lifting for the $\nu=1,2$, and 3 splittings originate from electron-electron interactions. The field dependence of the longitudinal resistance at the $\nu=0$ charge neutral point reveals insulating behavior similar in character to that of single layer graphene.

The authors thank D. Abanin, Y. Zhang, E. A. Henriksen, F. Ghahari, and Y. Barlas for helpful discussion, and thank S. T. Hannahs, E. C. Palm, and T. P. Murphy for their experimental assistance. This work is supported by DOE (No. DEFG02-05ER46215). A portion of this work was performed at the National High Magnetic Field Laboratory, which is supported by NSF Cooperative Agreement No. DMR-0654118, by the State of Florida, and by the DOE.

Note added.-During the preparation of this Letter, we became aware of a related work with similar conclusions by Feldman et al. [20].

[1] K. S. Novoselov et al., Nature (London) 438, 197 (2005).

[2] Y. Zhang, Y. Tan, H. L. Stormer, and P. Kim, Nature (London) 438, 201 (2005).

[3] K. S. Novoselov et al., Nature Phys. 2, 177 (2006).

[4] Edward McCann and Vladimir I. Fal'ko, Phys. Rev. Lett. 96, 086805 (2006).

[5] Yafis Barlas, R. Côté, K. Nomura, and A. H. MacDonald, Phys. Rev. Lett. 101, 097601 (2008).

[6] Y. Zhang et al., Phys. Rev. Lett. 96, 136806 (2006).

[7] Z. Jiang, Y. Zhang, H. L. Stormer, and P. Kim, Phys. Rev. Lett. 99, 106802 (2007).

[8] A. J. M. Giesbers et al., Phys. Rev. B 80, 201403(R) (2009).

[9] Z. Jiang, Y. Zhang, Y.-W. Tan, H. L. Stormer, and P. Kim, Solid State Commun. 143, 14 (2007).

[10] K. Yang, Solid State Commun. 143, 27 (2007).

[11] J. G. Checkelsky, L. Li, and N. P. Ong, Phys. Rev. Lett. 100, 206801 (2008).

[12] J. G. Checkelsky, L. Li, and N. P. Ong, Phys. Rev. B 79, 115434 (2009).

[13] K. Shizuya, Phys. Rev. B 79, 165402 (2009).

[14] D. A. Abanin, S.A. Parameswaran, and S. L. Sondhi, Phys. Rev. Lett. 103, 076802 (2009).

[15] R. Nandkishore and L. Levitov, arXiv:0907:5395v1.

[16] K. S. Novoselov et al., Proc. Natl. Acad. Sci. U.S.A. 102, 10451 (2005)

[17] E. V. Castro et al., Phys. Rev. Lett. 99, 216802 (2007).

[18] S. Adam and S. Das Sarma, Phys. Rev. B 77, 115436 (2008).

[19] S. Das Sarma and Kun Yang, Solid State Commun. 149, 1502 (2009).

[20] B. Feldman, J. Martin, and A. Yacoby, Nature Phys. 5, 889 (2009). 